\title{
CHRONIC ETHANOL INTAKE ALTERS CIRCADIAN PERIOD-RESPONSES TO BRIEF LIGHT PULSES IN RATS
}

\author{
Alan M. Rosenwasser, Ryan W. Logan, and Matthew E. Fecteau \\ Department of Psychology, University of Maine, Orono, ME, USA
}

\begin{abstract}
Although chronic alcohol intake is associated with widespread disruptions of sleep-wake cycles and other daily biological rhythms in both human alcoholics and experimental animals, the extent to which the chronobiological effects of alcohol are mediated by effects on the underlying circadian pacemaker remains unknown. Nevertheless, recent studies indicate that both adult and perinatal ethanol treatments may alter the free-running period and photic responsiveness of the circadian pacemaker. The present experiment was designed to further characterize the effects of chronic ethanol intake on the response of the rat circadian pacemaker to brief light pulses. Ethanol-treated and control animals were exposed to 15-min light pulses during either early or late subjective night on the first day of constant darkness following entrainment to a 12:12 light-dark cycle. Relative to pulses delivered during early subjective night and to "no-pulse" conditions, light pulses delivered during late subjective night resulted in period-shortening after-effects under constant darkness, but only in control animals, not in ethanol-treated animals. These results indicate that chronic ethanol intake reduces the responsiveness of the circadian pacemaker to acute photic stimulation, and suggest that the chronobiological disruptions seen in human alcoholics are due in part to alterations in circadian pacemaker function.
\end{abstract}

Keywords Circadian pacemaker, Alcohol, Light pulses, Free-running period, Rodent models

\section{INTRODUCTION}

Chronic alcohol intake is associated with widespread disruptions of sleep-wake cycles and of other daily biological rhythms in both human alcoholics (Sano et al., 1993; Fonzi et al., 1994; Schmidz et al., 1996; Mukai et al., 1998; Brower, 2001) and experimental animals (Rouhani et al., 1990; Rajakrishnan et al., 1999; Ehlers and Slawecki, 2000; El-Mas and Abdel-Rahman, 2000; Danel and Touitou, 2004). Despite these welldocumented disruptions, the extent to which the chronobiological effects of alcohol are mediated by effects on the underlying circadian pacemaker

Address correspondence to Alan M. Rosenwasser, Department of Psychology, University of Maine, 5742 Little Hall, Orono, ME 04469-5742, USA. E-mail: alanr@maine.edu 
is not known, primarily as a consequence of the historical lack of studies conducted under free-running conditions (Rosenwasser, 2001). Thus, although several ethanol-sensitive neurotransmitter and receptor systems are known to play key roles in circadian pacemaker regulation (Rosenwasser, 2001), the hypothesis that alcohol intake affects the circadian pacemaker via its action on these neural systems remains largely untested. This surprising lack of evidence stands in marked contrast to the extensive available data indicating that the central circadian pacemaker is indeed sensitive to several other classes of mood-altering psychoactive drugs, including antidepressants (Wollnik, 1992; Klemfuss and Kripke, 1994; Duncan et al., 1998), benzodiazepines (Turek and Losee-Olson, 1986; Subramanian and Subbaraj, 1996), and putative depressogenic agents (Rosenwasser, 1996).

Despite the development of analytical tools that effectively "unmask" the endogenous period and phase of the human circadian pacemaker (Czeisler, 1995), such approaches have not yet been applied to the analysis of alcohol-induced circadian rhythm disruptions in either normal or alcoholic subjects. On the other hand, emerging evidence from animal experiments suggests that both adult (Mistlberger and Nadeau, 1992; Dwyer and Rosenwasser, 1998; Rosenwasser et al., 2005) and perinatal (Earnest et al., 2001) ethanol treatment can modify the free-running circadian period, a fundamental parameter of the circadian pacemaker. Indeed, chronic ethanol treatment can evoke both lengthening and shortening of free-running circadian period (Rosenwasser et al., 2005), similar to the period-altering effects of chronic treatment with other mood-altering drugs (Rosenwasser, 1996; Subramanian and Subbaraj, 1996; Rosenwasser and Wirz-Justice, 1997).

In addition to their effects on free-running period, these same drug treatments also modify the response of the circadian pacemaker to light, including the acute phase-shifting effects of brief light pulses (Subramanian and Subbaraj, 1996; Duncan et al., 1998; Dwyer and Rosenwasser, 2000), the period-lengthening effect of constant light (Dwyer and Rosenwasser, 2000), and the phase of steady-state entrainment under daily light-dark cycles (Tamarkin et al., 1983). Thus, our study was designed to more completely characterize the chronobiological effects of ethanol by examining the effects of chronic ethanol treatment on the response of the circadian pacemaker to brief light pulses in rats.

\section{METHODS}

\section{Subjects and Apparatus}

Male Long-Evans rats were obtained from Charles River Laboratories (Wilmington, Massachusetts) and maintained individually in running- 
wheel cages (wheel diameter: $35 \mathrm{~cm}$; Lafayette Instruments, Lafayette, Indiana) with attached side cages. Running-wheel cages were placed within light- and sound-shielded enclosures equipped with exhaust fans and programmable lighting provided by incandescent lamps. Wheel revolutions were monitored via microswitches and a computer interface system (Dataquest III, MiniMitter Co., Bend, Oregon), and stored in 10-min blocks for subsequent analysis. Food and drinking fluid (either plain water or ethanol solution) were always freely available. The experiments were conducted following the guidelines of the Journal for the ethical study of biological rhythm phenomena of animal models (Touitou et al., 2004).

\section{Procedures}

This experiment utilized a between-groups design to compare the effects of brief light pulses on free-running rhythms in separate groups of ethanol-treated and control (water) animals ( $n=6$ per group). Animals in both groups were initially maintained under a light-dark cycle (LD 12:12), during which ethanol drinking solution was introduced gradually to the experimental group (5\% for 7 days, $10 \%$ for 8 days, and $20 \%$ thereafter). Weekly fluid intakes were recorded for animals in both groups throughout the course of the experiment. After introduction of the $20 \%$ ethanol solution to the experimental animals, animals in both groups were subsequently maintained under alternating epochs of LD 12:12 and constant darkness (DD) (LD1, 23 days; DD 1, 12 days; LD2, 16 days; DD2, 15 days; LD3, 16 days; DD3, 15 days; LD4, 21 days, DD4, 15 days). Brief light pulses (15 min, approximately 50 lux) were administered on the first complete day of DD1, DD2, and DD3; as a control procedure, no light pulses were administered at the beginning of DD4. Pulses were delivered at either Zeitgeber Time (ZT) 14 (DD1) or ZT 21 (DD2 and DD3). By convention, ZT 14 refers to a time $2 \mathrm{~h}$ after the projected time of lights-off, while ZT 21 refers to $9 \mathrm{~h}$ after projected lights-off. Thus, in this experiment, light pulses were delivered after either 26 (ZT 14) or 35 (ZT 21) h of darkness. This procedure is a form of the so-called "Aschoff Type-2" protocol, designed to ensure that animals are stably entrained to the LD cycle at the beginning of each DD test phase, and commonly used for assessment of both photic and nonphotic phase-shifting stimuli. Although this experiment was originally designed to assess the acute phase-shifting effects of the light pulses, difficulties in reliably determining the phase of rat activity onsets across days, coupled with the use of relatively brief DD exposures, made this impractical. Instead, we assessed the effects of the light pulses on the free-running circadian period as expressed during the subsequent DD epoch, as described below. 


\section{Data Analysis}

The free-running circadian period was determined for each DD epoch using three widely employed and complementary approaches: cosinor spectral analysis, $\chi^{2}$ periodogram analysis, and visual inspection. Cosinor analysis was performed using the routine included with the Dataquest program, while periodogram analysis was performed using the routine included in the Tau software package (MiniMitter, Bend, Oregon). Visual-graphical estimates of free-running period were obtained with the assistance of an automated procedure implemented in the Tau program. The period estimates obtained by the three procedures were generally in good agreement, and in fact, preliminary statistical analyses produced similar overall findings for each of the three measures considered separately. Thus, to minimize the variance of period estimates, the three measures were averaged to derive the period values reported in this paper. These values were subjected to a $2 \times 4$ two-factor repeatedmeasures ANOVA (group: ethanol-treated, control; DD test trial: DD1, DD2, DD3, DD4), followed by post-hoc tests for simple main effects as appropriate.

\section{RESULTS}

Figure 1 presents selected excerpts from the locomotor activity records of one control and one ethanol-treated animal, illustrating typical responses to light pulses presented at ZT 14 (LD1, DD1) and ZT 21 (LD2, DD2). Representative of all animals in this experiment, these records demonstrate relatively stable steady-state entrainment under LD and free-running circadian rhythmicity under DD. While both animals showed free-running periods in DD very close to $24 \mathrm{~h}$ following the ZT 14 light pulse, light pulses delivered at ZT 21 generally resulted in shorter, $<24 \mathrm{~h}$ periods under DD. Further, free-running periods following ZT 21 light pulses were typically shorter in control than in ethanol-treated animals, in both DD2 and DD3.

Mean ( + SEM) free-running periods for both groups across the four different DD test trials are presented in Figure 2. As suggested by inspection of the activity records (Figure 1), ANOVA revealed a significant main effect of test trial $(p=0.0001)$, indicating that free-running periods differed across the different light-pulse testing conditions, and a significant group-by-test trial interaction $(p=0.004)$, indicating that ethanol-treated and control animals differed in their response to the different light pulses. As a follow-up analysis, separate one-factor (test trial) ANOVAs were computed for each group, revealing a significant effect of test trial on the free-running period in control $(p=0.0001)$ but not in ethanoltreated animals. Finally, separate pairwise comparison of the two groups 

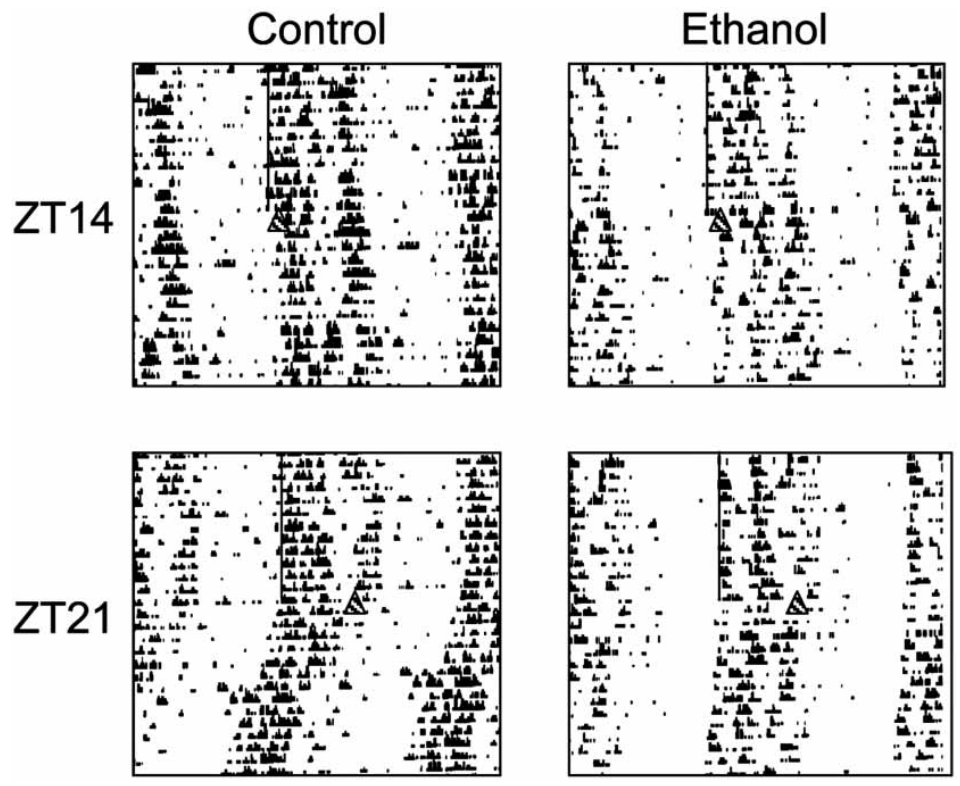

FIGURE 1 Segments of double-plotted (48 h span) activity records from one control (left) and one ethanol-treated (right) animal, illustrating typical responses to light pulses delivered at either Zeitgeber Time (ZT) 14 (top) or ZT 21 (bottom), on the first complete day of constant DD following entrainment to a 12:12 LD cycle. Superimposed vertical lines show the onset of darkness under the LD cycle, and the triangular symbols show the times of the light pulses. Both animals displayed similar free-running periods in constant DD following the ZT 14 light pulse, but the control animals displayed a marked period-shortening aftereffect relative to the ethanol-treated animal following the ZT 21 light pulse.

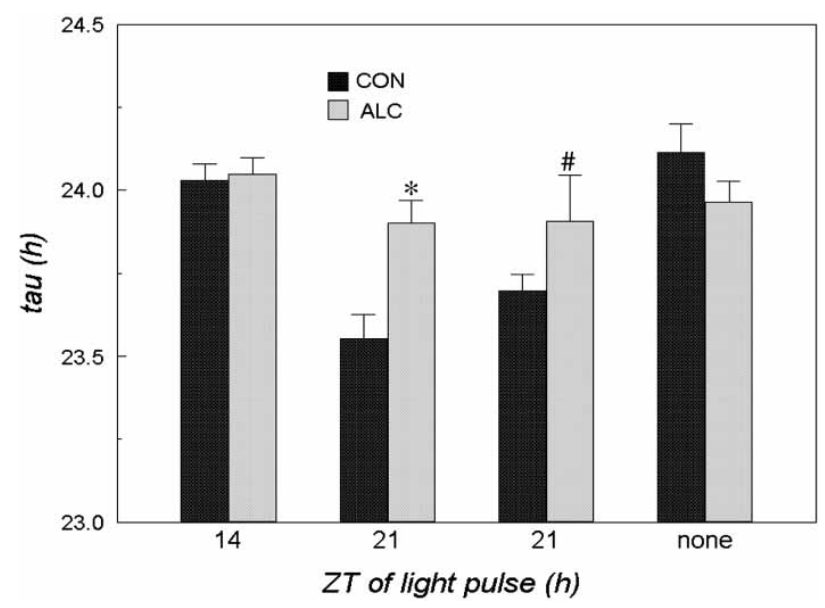

FIGURE 2 Mean ( + SEM) free-running period under constant darkness (DD) for the Zeitgeber Time (ZT) 14 (DD1), both ZT 21 (DD2 and DD3), and no-pulse (DD4) conditions. Control animals (CON; black bars) displayed significant shortening of free-running period following ZT 21 light pulses, but ethanol-treated animals (ALC; gray bars) did not. ${ }^{*}, p=0.005$; $\#, p=0.07$. See text for additional statistical analyses. 
for each test trial showed a significant group difference for trial 2 $(p=0.005)$ and a near-significant difference for trial $3(p=0.07)$. To summarize, light pulses presented at ZT 21 resulted in shortening of the free-running circadian period in DD relative to either ZT 14 light pulses or to no-pulse conditions, but only for control animals.

Ethanol-treated animals consumed significantly less fluid than did control animals over the course of exposure to $20 \%$ ethanol drinking solution (controls: $46.82 \mathrm{~mL} /$ day; ethanol-treated: $24.84 \mathrm{~mL} /$ day, $p=0.001$ ). While we cannot rule out a possible contribution of dehydration to the effects described here, there were no apparent differences in general body condition of the two groups at the end of the experiment, and we previously found no differences in body weight growth curves between similarly treated ethanol-maintained and control rats housed under standard colony conditions (unpublished observations). Ethanoltreated animals in this experiment ingested an average of $3.93 \mathrm{~g} /$ day ethanol, and based on body weight curves measured in our colonymaintained animals, we can estimate that the treated animals in the present study consumed about $8 \mathrm{~g} / \mathrm{kg} /$ day ethanol.

Previous research (Hiller-Sturmhofel and Kulkosky, 2001; Rosenwasser et al. unpublished observations) indicates that under conditions of $24 \mathrm{~h}$ access, rats consume ethanol solutions in a series of small bouts, closely resembling the normal nocturnal pattern of water intake. Based on published work, the level of ethanol intake achieved in this study would be expected to produce maximal blood ethanol levels of about 50 to $100 \mathrm{mg} /$ dL during early subjective night, with near-zero levels during most of the circadian daytime (Aalto, 1986; Murphy et al., 2002). At no time did we observe any gross differences in the behavior of ethanol-treated and control animals over the course of this study.

\section{DISCUSSION}

The results of this experiment indicate that chronic ethanol intake alters the response of the rat circadian pacemaker to brief light pulses. While the effects of brief light pulses on the circadian pacemaker have usually been evaluated with respect to their ability to evoke acute circadian phase shifting, this study focused instead on the longer-lived "after-effects" of brief light pulses on the free-running circadian period. In fact, it has long been recognized that acute perturbation of the circadian pacemaker can induce long-lasting period aftereffects, such that light pulses delivered during early subjective night result in both an acute phase-delay as well as long-term period-lengthening, while light pulses delivered during late subjective night result in an acute phase-advance accompanied by long-term period-shortening (Pittendrigh and Daan, 1976). Indeed, recent analyses have emphasized the potential importance 
of such period-responses in ensuring stable entrainment under natural conditions (Beersma et al., 1999; Daan, 2000; Daan and Aschoff, 2001; Sharma and Daan, 2002). In the present study, we analyzed periodresponses to light pulses rather than phase-responses because they proved to be more reliably quantifiable under our experimental conditions, and as expected, we found significant period-shortening after late-night (ZT 21) light pulses in control animals. In contrast, since control animals did not display differences in free-running period following early-night (ZT 14) light pulses relative to no-pulse conditions, we conclude that the early-night pulses employed in this study did not evoke period aftereffects. Thus, while the present results indicate that chronic ethanol treatment blocks period after-effects resulting from latenight light pulses, they are inconclusive with regard to whether such an effect would also be seen for effective early-night light pulses.

In general, the effects of chronic ethanol treatment on free-running circadian rhythms appear quite similar to those of other mood-altering drugs. For example, alterations in free-running circadian period have been described during chronic administration of several different classes of antidepressants (Wollnik, 1992; Klemfuss and Kripke, 1994; Rosenwasser and Wirz-Justice, 1997; Duncan et al., 1998), the anxiolytic benzodiazepine, diazepam (Subramanian and Subbaraj, 1996), the putative depressogenic agent, clonidine (Rosenwasser, 1996), and ethanol (Dwyer and Rosenwasser, 1998; Rosenwasser et al., 2005). Surprisingly, both lengthening and shortening of free-running period have been observed, even for a given agent, and these effects appear to be modulated by a variety of factors, including both lighting conditions and individual differences in baseline period (Rosenwasser, 1996; Rosenwasser et al., 2005). In contrast, the reported effects of mood-altering drugs on the response of the circadian pacemaker to light pulses have been more consistent: despite their diverse pharmacological effects, clorgyline, lithium (Duncan et al., 1998), diazepam (Subramanian and Subbaraj, 1996), clonidine (Dwyer and Rosenwasser, 2000), and ethanol (present data) all appear to blunt the pacemaker's responsiveness to light. In addition, two laboratories have recently reported blunting of the circadian pacemaker's response to photic stimuli in adulthood following perinatal ethanol treatments (Sei et al., 2003; Farnell et al., 2004).

Taken together with our previous findings that chronic ethanol intake alters circadian period during long-term maintenance in free-running conditions (Dwyer and Rosenwasser, 1998; Rosenwasser et al., 2005), the present observations indicate that the chronobiological effects of ethanol are due, at least in part, to alterations at the level of the underlying circadian pacemaker. Unlike effects of ethanol on circadian phase, amplitude and waveform under steady-state entrainment that have been amply documented in both human alcoholics and experimental animals, it is 
widely accepted that the effects of a drug (or other stimulus) on the expressed free-running circadian period must reflect changes in the period of the circadian pacemaker, either as a result of direct pharmacological interaction with pacemaker neurons, or via action on one or more of the pacemaker's input (entrainment) pathways. Thus, while the present results demonstrate that chronic ethanol intake alters circadian pacemaker function, they do not prove that ethanol exerts these effects via direct interaction with the hypothalamic suprachiasmatic nucleus $(\mathrm{SCN})$, known to be the site of the primary mammalian pacemaker (Rosenwasser, 2003).

While the specific sites and mechanisms by which ethanol influences the circadian pacemaker are unknown, it is clear that several ethanolsensitive neurotransmitter systems participate in circadian pacemaker regulation. Thus, chronic ethanol treatment reduces the expression of several neuropeptides within the SCN (Madeira et al., 1997). In addition, serotonin interacts bidirectionally with ethanol intake (LeMarquand et al., 1994), and a robust midbrain serotonergic projection to the SCN modulates both the free-running period and light response of the SCN circadian pacemaker (Rosenwasser, 2003). Similarly, the neurobehavioral effects of ethanol are mediated partially via indirect agonist effects on the gamma-aminobutyric acid (GABA)-A receptor (Faingold et al., 1998), and GABA-A receptors on SCN pacemaker neurons modulate both circadian period and photic responsiveness (Rosenwasser, 2003). Finally, ethanol also evokes indirect antagonist effects at the NMDA-type glutamate receptor (Faingold et al., 1998), which plays a critical role in transducing photic signals arising from the direct retinal-hypothalamic tract innervating the SCN (Rosenwasser, 2003). Further identification of the mechanisms by which ethanol influences the circadian pacemaker could eventually prove useful in the management of sleep and other chronobiological disruptions seen commonly in human alcoholics.

\section{REFERENCES}

Aalto, J. (1986). Circadian drinking rhythms and blood alcohol levels in two rat lines developed for their alcohol consumption. Alcohol 3:73-75.

Beersma, D.G.M., Daan, S., Hut, R.A. (1999). Accuracy of natural entrainment under fluctuating light conditions: contributions of phase and period responses. J. Biol. Rhythms 14:320-329.

Brower, K.J. (2001). Alcohol's effects on sleep in alcoholics. Alc. Res. Health 25:110-125.

Czeisler, C.A. (1995). The effect of light on the human circadian pacemaker. In: Waterhouse, J.M., ed. Circadian Clocks and Their Adjustment. Chichester: Wiley, pp. 254-302.

Daan, S., Aschoff, J. (2001). The entrainment of circadian systems. In: Takahashi, J.S., Turek, F.W., Moore, R.Y., eds. Handbook of Behavoral Neurobiology, Vol. 12: Circadian Clocks. New York: Kluwer Academic/Plenum Publishers, pp. 7-43.

Danel, T., Touitou, Y. (2004). Chronobiology of alcohol: from chronokinetics to alcohol-related alterations of the circadian system. Chronobiol. Int. 21:923-935.

Dwyer, S.M., Rosenwasser, A.M. (1998). Neonatal clomipramine treatment, alcohol intake and circadian rhythms in rats. Psychopharmacol. 138:176-183. 
Dwyer, S.M., Rosenwasser, A.M. (2000). A noradrenergic mechanism influences the circadian timing system in rats and hamsters. Biol. Rhythm Res. 31:355-373.

Earnest, D.J., Chen, W-J.A., West, J.R. (2001). Developmental alcohol and circadian clock function. Alc. Res. Health 25:136-140.

Ehlers, C.L., Slawecki, C.J. (2000). Effects of chronic ethanol exposure on sleep in rats. Alcohol 20: $173-179$.

El-Mas, M.M., Abdel-Rahman, A.A. (2000). Radiotelemetric evaluation of hemodynamic effects of long-term ethanol in spontaneously hypertensive and wistar-kyoto rats. J. Pharmacol. Exp. Therapeut. 292:944-951.

Faingold, C.L., N'Gouemo, P., Riaz, A. (1998). Ethanol and neurotransmitter interactions-from molecular to integrative effects. Prog. Neurobiol. 55:509-535.

Farnell, Y.Z., West, J.R., Chen, W.-J.A., Allen, G.C., Earnest, D.J. (2004). Developmental alcohol exposure alters light-induced phase shifts of the circadian activity rhythm in rats. Alcohol: Clin. Exp. Res. 28:1020-1027.

Fonzi, S., Solinas, G.P., Costelli, P., Parodi, C., Murialdo, G., Bo, P., Albergati, A., Montalbetti, L., Savoldi, F., Polleri, A. (1994). Melatonin and cortisol circadian secretion during ethanol withdrawal in chronic alcoholics. Chronobiologia 21:109-112.

Hiller-Sturmhofel, S., Kulkosky, P. (2001). Chronobiological regulation of alcohol intake. Alc. Res. Health 25:141-148.

Kakihana, R., Moore, J.A. (1976). Circadian rhythm of corticosterone in mice: effect of chronic consumption of alcohol. Psychopharmacologia 46:301-305.

Klemfuss, H., Kripke, D.F. (1994). Antidepressant and depressogenic drugs lack consistent effects on hamster circadian rhythms. Psych. Res. 53:173-184.

LeMarquand, D., Pihl, R.O., Benkelfat, C. (1994). Serotonin and alcohol intake, abuse, and dependence: findings of animal studies. Biol. Psych. 36:395-421.

Madeira, M.D., Andrade, J.P., Lieberman, A.R., Sousa, N., Almeida, O.F.X., Paula-Barbosa, M.M. (1997). Chronic alcohol consumption and withdrawal do not induce cell death in the suprachiasmatic nucleus, but lead to irreversible depression of peptide immunoreactivity and mRNA levels. J. Neurosci. 17:1302-1319.

Mistlberger, R.E., Nadeau, J. (1992). Ethanol and circadian rhythms in the syrian hamster: effects on entrained phase, reentrainment rate, and period. Pharmacol. Biochem. Behav. 43:159-165.

Mukai, M., Uchimura, N., Hirano, T., Ohshima, H., Ohshima, M., Nakamura, J. (1998). Circadian rhythms of hormone concentrations in alcohol withdrawal. Psych. Clin. Neurosci. 52:238-240.

Murphy, J.M., Stewart, R.B., Bell, R.L., Badia-Elder, N.E., Carr, L.G., McBride, W.J., Lumeng, L., Li, T.-K. (2002). Phenotypic and genotypic characterization of the Indiana university rat lines selectively bred for high and low alcohol preference. Behav. Genetics 32:363-388.

Pittendrigh, C.S., Daan, S. (1976). Functional analysis of circadian pacemakers in nocturnal rodents. I. The stability and lability of spontaneous frequency. J. Comp. Physiol. 106:223-252.

Rajakrishnan, V., Subramanian, P., Viswanathan, P., Menon, V.P. (1999). Effect of chronic ethanol ingestion on biochemical circadian rhythms in Wistar rats. Alcohol 18:147-152.

Rouhani, S., Emmanouilidis, E., Tran, G., Durlach, J., Paya, C., Fermanian, J., Manicom, R., Soulairac, A., Poenaru, S. (1990). Circadian variations in vigilance states in the alcohol-dependent rat. Physiol. Behav. 48:637-640.

Rosenwasser, A.M. (1996). Clonidine shortens free-running circadian period in both constant light and constant darkness. Physiol. Behav. 60:373-379.

Rosenwasser, A.M. (2001). Alcohol, antidepressants and circadian rhythms: human and animal models. Alc. Res. Health 25:126-135.

Rosenwasser, A.M. (2003). Neurobiology of the mammalian circadian system: oscillators, pacemakers, and pathways. In: Fluharty, F.J., Grill, H.J., eds. Progress in Psychobiology and Physiological Psychology, Vol. 18. San Diego: Elsevier Academic Press, pp. 1-38.

Rosenwasser, A.M., Wirz-Justice, A. (1997). Circadian rhythms and depression: clinical and experimental models. In: Redfern, P.H., Lemmer, B., eds. Physiology and Pharmacology of Biological Rhythms. Berlin: Springer Verlag, pp. 457-486.

Rosenwasser, A.M., Fecteau, M.E., Logan, R.W. (2005). Effects of ethanol intake and ethanol withdrawal on free-running circadian activity rhythms in rats. Physiol. Behav. in press.

Sano, H., Suzuki, Y., Yazaki, R., Tamefusa, K., Ohara, K., Yokoyama, T., Miyasato, K., Ohara, K. (1993). Circadian variation in plasma 5-hydroxyindoleacetic acid level during and after alcohol 
withdrawal: phase advances in alcoholic patients compared with normal subjects. Acta. Psychiat. Scand. 87:291-296.

Schmitz, M.M., Sepandj, A., Pichler, P.M., Rudas, S. (1996). Disrupted melatonin secretion during alcohol withdrawal. Prog. Neuro-Psychopharm. Biol. Psychiat. 20:983-995.

Sei, H., Sakata-Haga, H., Ohta, K., Sawada, K., Morita, Y., Fukui, Y. (2003). Prenatal exposure to alcohol alters the light response in postnatal circadian rhythm. Brain Res. 987:131-134.

Sharma, V.J., Daan, S. (2002). Circadian phase and period responses to light stimuli in two nocturnal rodents. Chronobiol. Int. 19:659-670.

Subramanian, P., Subbaraj, R. (1996). Diazepam modulates the period of locomotor rhythm in mice (Mus booduga) and attenuates light-induced phase advances. Pharmacol. Biochem. Behav. 54: 393-398.

Tamarkin, L., Craig, C., Garrick, N., Wehr, T. (1983). Effect of clorgyline (a MAO type A inhibitor) on locomotor activity in the Syrian hamster. Am.J. Physiol. 245:R215-R221.

Touitou, Y., Poraluppi, F., Smolensky, M.H., Rensing, L. (2004). Ethical principles and standards for the conduct of human and animal biological rhythm research. Chronobiol. Int. 21:161-170.

Wollnik, F. (1992). Effects of chronic administration and withdrawal of antidepressant agents on circadian activity rhythms in rats. Pharmacol. Biochem. Behav. 43:549-561. 